\title{
Novel dermo-epidermal equivalents on silk fibroin-based formic acid-crosslinked three-dimensional nonwoven devices with prospective applications in human tissue engineering/regeneration/repair
}

\author{
ILARIA DAL PRA ${ }^{1}$, ANNA CHIARINI ${ }^{1}$, ALESSANDRA BOSCHI ${ }^{2}$, \\ GIULIANO FREDDI ${ }^{2}$ and UBALDO ARMATO ${ }^{1}$ \\ ${ }^{1}$ Histology \& Embryology Unit, Department of Biomedical \& Surgical Sciences, \\ University of Verona Medical School, Verona; ${ }^{2}$ Silk Research Institute, Milan, Italy
}

Received March 8, 2006; Accepted April 28, 2006

\begin{abstract}
Biologically well-tolerated materials are at the core of intense research. In this study, we report both the intrinsic features of formic acid (FA)-crosslinked threedimensional (3D) nonwoven scaffolds consisting of sericindeprived, Bombyx mori silk fibroin (SF) in $\beta$-sheet form and the long-term co-culture of adult human epidermal keratinocytes (HEKs) and dermal fibroblasts (HDFs) on them. The results of scanning electron microscopy, differential scanning calorimetry, and thermogravimetric and tensile strength studies showed that such scaffolds are made by a composite material, in which anisotropic SF fibres are enclosed within an isotropic matrix of SF in film form. Both fibres and films are firmly crosslinked by FA treatment and water-insoluble owing to their $\beta$-sheet crystalline structure. Moreover, while a certain strength of the material in the dry state may favour its manufacturing, handling, and shaping, the dramatic loss of hardiness under wet conditions renders the scaffolds softer, mechanically more compliant and, hence, more apt for implantation. Normal HEKs and HDFs could be successfully co-cultured on such nonwovens for up to 75-95 days in vitro, thus forming a novel kind of dermo-epidermal equivalent, in
\end{abstract}

Correspondence to: Professor Ubaldo Armato, Histology and Embryology Unit, Department of Biomedical and Surgical Sciences, University of Verona Medical School, Strada Le Grazie 8, Verona I-37134, Italy

E-mail: ubaldo.armato@univr.it

Abbreviations: 3D, three-dimensional; AHKF, co-culture set of adult human keratinocytes and fibroblasts; DSC, differential scanning calorimetry; FA, formic acid; FBR, foreign body response; HDFs, (adult) human dermal fibroblasts; HEKs, (adult) human epidermal keratinocytes; IL-1ß, interleukin-1ß; SEM, scanning electron microscopy; SF, Bombyx mori silk fibroin; TGA, thermogravimetric analysis

Key words: silk fibroin, epidermal keratinocytes, dermal fibroblasts which the cells were metabolically active and performed specific functions (e.g. the de novo production and assembly of collagen fibres), but never released urea nitrogen (an index of protein catabolism) or proinflammatory interleukin-1ß (IL-1ß). In keeping with the results of previous studies in animals (Dal Pra I, et al, Biomaterials 26: 1987-1989, 2005), these findings support the view that 3D SF-based nonwovens may be excellent candidates for beneficial applications in the field of human tissue engineering/regeneration/repair.

\section{Introduction}

Raw silk fibres are composed of inner filaments of a protein, fibroin, embedded in an outer rubbery coating formed by another protein, i.e. sericin. Silk fibroin (SF) is a linear polypeptide mainly containing non-polar amino acids such as glycine and alanine (1). Silk threads were used for surgical stitches until sensitization to sericin, revealed by type-I allergic responses such as asthma and upregulated levels of specific IgEs, was reported to occur in patients repeatedly submitted to surgery (2-5). Conversely, surgical sutures performed with degummed, i.e. sericin-deprived, but wax- or silicone-coated (so called black braided) silk were found to be unable to induce sensitization responses in humans $(2,4)$. However, a moderate [according to the criteria of Setzen et al (6)] foreign body response (FBR) surrounded black-braided silk sutures persisting longer than one month (7). In addition, at variance with black braided silk, regenerated SF elicited no thrombogenicity in vivo (8), while in vitro studies revealed that SF had no proinflammatory properties (9-11), and that SF films could support the adhesion and growth of rodent fibroblasts (12-16) and several types of normal adult human cells, including keratinocytes, fibroblasts, osteoblasts, endothelial cells, and astrocytes $(10,11,17,18)$. Due to its ability to promote cell adhesion and growth, SF has been the object of increasing interest as a potential biomaterial forming the core or coating the surfaces of scaffolds aimed at tissue engineering/ regeneration/repair purposes $(11-13,19)$. Yet, information on the in vivo long-term biocompatibility of SF scaffolds, on their induction of local tissue reactions, and on their ability to 
efficiently favour tissue engineering/regeneration/repair is scanty. In a previous study, we investigated some of the structural features of novel SF-based FA-crosslinked 3D nonwoven scaffolds and the long-term effects of their implantation into the subcutaneous tissue of C57BL6 mice. Thus, we found that in the course of six months these nonwovens were well tolerated as they elicited only a quite mild FBR with no fibrogenicity while concurrently directing the de novo production of a vascularized reticular connective tissue both in the interstices within and onto their fibres (20). In the present study, we have further explored the physical, structural, and human biocompatibility properties of such SF-based FAcrosslinked nonwovens showing, amongst other things, that normal adult HEKs and HDFs can be successfully co-cultured on the anastomising SF fibres of such scaffolds for rather lengthy terms. Hence, the whole of these results suggests that, once produced and structurally fashioned (and even improved) ad hoc, these 3D nonwoven scaffolds may find beneficial applications for tissue engineering/regeneration/repair purposes in various clinical settings.

\section{Materials and methods}

Production of SF-based FA-crosslinked 3D nonwovens. Raw silk fibres from Bombyx mori cocoons were boiled for $1 \mathrm{~h}$ in a $0.7 \%$ w/v soap solution, and then rinsed thoroughly with water to remove sericin, a protein gum surrounding the fibroin filaments. After drying at room temperature, the degummed silk fibres were extracted with diethyl ether to remove residual fatty acids. 3D SF-based nonwovens were prepared according to the method of Armato et al (21). Briefly, degummed silk fibres were cut in short snippets and soaked at room temperature into $98 \%$ FA containing $0.01 \%{ }^{\mathrm{w} / \mathrm{v}} \mathrm{CaCl}_{2}$ (material-tofluid ratio 1:200). The fibre suspension was shaken for approximately $30 \mathrm{~min}$ to achieve homogeneous fibre distribution. The acidic solution was evaporated under atmospheric conditions, and the resulting nonwoven was repeatedly washed with double distilled water to remove any residual salt and finally vacuum dried. For cell culture studies, the scaffolds were sterilised by a 30-min exposure of each of their two main surfaces to ultraviolet rays (UV) emitted by bactericidal lamps hanging from the roof of a closed tin box $(30 \times 30 \times 50 \mathrm{~cm}$ in size) at room temperature. Next, they were cut into $2.7 \times 2.9-\mathrm{cm}$ pieces with sterile scalpels, and the cut nonwoven pieces were carefully placed inside the wells of 8-well multidish plates (Nunc Ltd.) containing $0.4 \mathrm{ml}$ of Dulbecco's modified Eagle's minimum essential medium (DMEM; Sigma) to prevent them from drying.

Scanning electron microscopy of SF-based FA-crosslinked $3 D$ non-wovens. The morphological characterization of 3D-SF nonwovens was performed by means of scanning electron microscopy (SEM), using a Stereoscan 440 microscope (LEO Electronic Microscopy Ltd.). Samples were observed at $10 \mathrm{kV}$ acceleration voltage, after gold coating under reduced argon atmosphere with a Med 020 coating system (BAL-TEC).

Differential scanning calorimetry (DSC) of SF-based FAcrosslinked $3 D$ non-wovens. DSC measurements were performed with a DSC-30 instrument (Mettler Toledo), from room temperature to $550^{\circ} \mathrm{C}$, at a heating rate of $10^{\circ} \mathrm{C} / \mathrm{min}$, on 3-mg samples. The open aluminium pan was swept with $\mathrm{N}_{2}$ during the analysis.

Thermogravimetric analysis (TGA) of SF-based FAcrosslinked $3 D$ nonwovens. TGA was performed with a TGA Q500 instrument (TA Instruments), from room temperature to $550^{\circ} \mathrm{C}$, at a heating rate of $20^{\circ} \mathrm{C} / \mathrm{min}$, on 3-mg samples. The cell was swept with $\mathrm{N}_{2}$ during the analysis.

Mechanical properties of SF-based FA-crosslinked 3D-SF nonwovens. Mechanical properties of 3D-SF nonwovens were measured on strips of $25 \times 2 \mathrm{~mm}(0.19-\mathrm{mm}$ thick) by means of an Instron tensile testing machine mod. 4501, at 10 -mm gauge length and $0.2 \mathrm{~mm} / \mathrm{min}$ crossbar rate, corresponding to a strain rate of $3.3 \times 10^{-4} \mathrm{~s}^{-1}$. Measurements were performed under standard conditions of temperature $\left(20^{\circ} \mathrm{C}\right)$ and humidity $(65 \%)$ on dried and wet samples. The latter were soaked in distilled water for $2 \mathrm{~h}$ at $37^{\circ} \mathrm{C}$ before measurement. Breaking strength, elongation at break, modulus, and energy were calculated from the stress-strain curves as the average of 5 measurements.

Human skin cell cultures on SF-based FA-crosslinked 3D-SF nonwovens. Two strains of normal adult HDFs were isolated from as many healthy volunteers, after informed consent was received. HDFs were cultured at $37^{\circ} \mathrm{C}$ in $90 \%$ v/v Dulbecco's modified Eagle's minimum essential medium (DMEM; Sigma), fortified with inactivated (at $56^{\circ} \mathrm{C}$ for $30 \mathrm{~min}$ ) foetal bovine serum (FBS, $10 \%$ v/v ; Biowhittaker $\mathrm{GmbH}$ ), and antibiotics. Upon reaching $75 \%$ confluence, HDFs were detached via a light treatment with ethylene glycol-bis (2aminoethylether)-N,N,N',N'-tetraacetic acid (EGTA)/trypsin solution, and their numbers and viable fractions were assessed. Prior to seeding onto the previously sterilised nonwoven pieces, fibroblasts were detached from the flasks and then labelled with 3,3'-dioctadecyloxacarbocyanine perchlorate $\left[\mathrm{DiOC}_{18}(3)\right.$; Molecular Probes Inc.], an intra-vital membrane phospholipid-binding probe emitting under appropriate UV light excitation a bright green fluorescence detectable with an optical FITC filter set. Next, $\sim 10^{6} \mathrm{HDFs}$ suspended in $1 \mathrm{ml}$ growth medium were slowly seeded onto the sterile pieces of 3D SF non-wovens. The specimens were incubated at $37^{\circ} \mathrm{C}$ for $3 \mathrm{~h}$ in an atmosphere of air with added $\mathrm{CO}_{2}(5 \%$ v/v $)$. Thereafter, the cell-bearing nonwovens were transferred to a second multi-dish plate, dipped into $2 \mathrm{ml}$ growth medium, and placed in the incubator at $37^{\circ} \mathrm{C}$ in air $95 \% / \mathrm{CO}_{2} 5 \%$ v/v The nonwovens were periodically checked by phase contrast or fluorescence optics. Spent medium was changed with fresh every 2-3 days. Cell-conditioned medium samples were stored at $-80^{\circ} \mathrm{C}$ for further analysis.

Normal adult HEKs were isolated via dispase digestion from 3 intra-operative biopsies taken, after informed consent, from 3 patients. HEKs were first seeded onto a feeder-layer of previously irradiated HDFs $\left(6 \times 10^{3}\right.$ rads $)$ and incubated in a mixture of 1:1 $1^{\mathrm{v} / \mathrm{v}}$ MCDB 153 medium (Sigma) and Ham's F12 medium (Biowhittaker $\mathrm{GmbH}$ ) with added $10 \%$ v/v inactivated FBS (Biowhittaker $\mathrm{GmbH}$ ). Upon reaching 75\% confluence, HEKs were detached from the flasks and then 
labelled with 1,1'-dioctadecyl-3,3,3,3'-tetramethyl-indocarbocyanine perchlorate [DilC ${ }_{18}(3)$; Molecular Probes Inc.], an intra-vital membrane phospholipid-binding probe emitting under proper UV light excitation a bright orange-red fluorescence discernible with a rhodamine optical filter set. Next, $\sim 10^{6}$ HEKs suspended in $1 \mathrm{ml}$ growth medium were seeded onto the surface of the SF 3D nonwovens opposite to that which had already been carrying the attached HDFs during the previous 10 days. The specimens were incubated at $37^{\circ} \mathrm{C}$ for $3 \mathrm{~h}$ in an atmosphere of air with added $\mathrm{CO}_{2} 5 \%$ v/v . Thereafter, the HDFs/HEKs-bearing nonwovens were transferred to a second multi-dish plate, dipped into $2 \mathrm{ml}$ growth medium for the HEKs described and placed in the incubator at $37^{\circ} \mathrm{C}$ in air $95 \% / \mathrm{CO}_{2} 5 \% \mathrm{v} / \mathrm{v}$. Spent growth media were changed with fresh every 2-3 days and samples of the cellconditioned media were stored at $-80^{\circ} \mathrm{C}$ for further analysis. The co-cultured cells were examined under an inverted Zeiss IM35 microscope and photographed live with an Olympus $3300^{\mathrm{TM}}$ digital camera. Deconvolved fluorescence images were obtained by using Huygens professional software for Windows (Scientific Volume Imaging b. v., Hilversun, The Netherlands).

Metabolic studies. In the cell-conditioned growth medium samples, glucose uptake and lactate release were evaluated via glucose oxidase and lactate oxidase assays (Sigma), respectively, while L-glutamine uptake was assessed by the decrease in absorbance at $340 \mathrm{~nm}$ due to the oxidation of NADPH. The results were expressed as cumulative values in $\mathrm{mg} / \mathrm{ml}$ of sample. The urea nitrogen content of cell-conditioned medium samples was assayed using the BUN (Endpoint) reagent (Sigma diagnostics procedure no. 66-UV revised January 1995) for quantitative enzymatic determinations of urea nitrogen concentrations in serum or plasma at $340 \mathrm{~nm}$ according to the instructions of the seller.

Release of proinflammatory IL-1 $\beta$ by ELISA assay. A commercial ELISA kit was used to assay the content of human IL-1B (CLB, Amsterdam, The Netherlands) in the conditioned co-culture media. The sensitivity of this assay was $0.8 \mathrm{pg} / \mathrm{ml}$.

Extracellular assembly of collagen fibres. The rate of type I collagen fibre assembly was assessed by an EIA method (Takara Shuzo Co., Japan) measuring the amount of Ctelopeptides released in the process from procollagen type-I molecules. The sensitivity of this assay was $10 \mathrm{ng} / \mathrm{ml}$.

Statistical analysis. The one-way analysis of co-variance was applied to sets of corresponding cell culture metabolism time-related data electing as the significance level $\mathrm{P}<0.05$.

\section{Results and discussion}

Characterization of 3D SF-based FA-crosslinked nonwovens. SEM photographs of the nonwovens are shown in Fig. 1. The low magnification image shows that the nonwoven consists of randomly arranged SF fibres of $12-14 \mu \mathrm{m}$ in width, which form a network characterised by the presence of many interconnected voids (Fig. 1a). At a higher magnification it

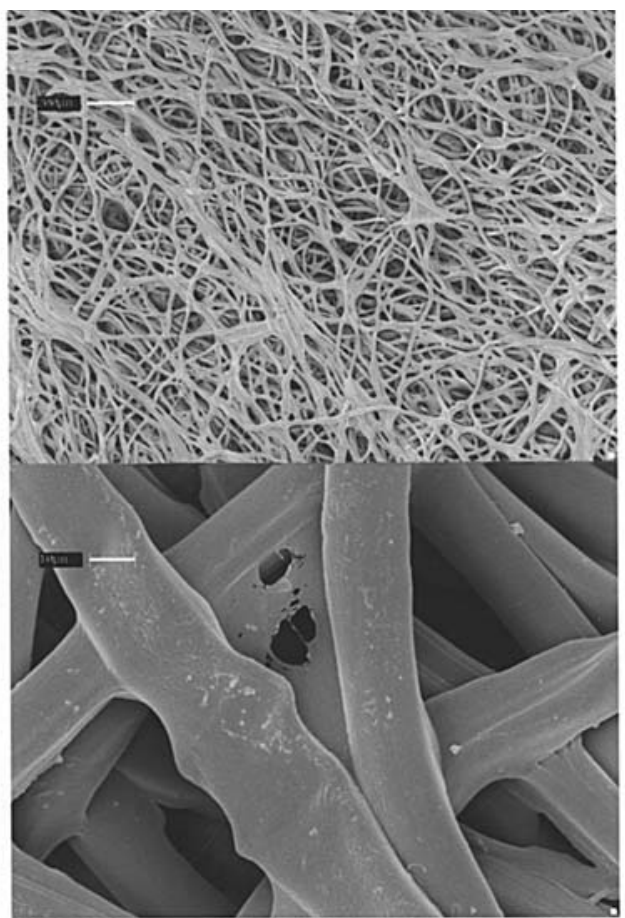

(a)

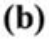

Figure 1. SEM photographs of 3D SF-based FA-crosslinked nonwovens. (a) Low magnification view of the surface showing the random arrangement of SF fibres. (b) Detail of the surface showing fibres stuck at crossovers and a thin film covering a void between adjacent fibres.

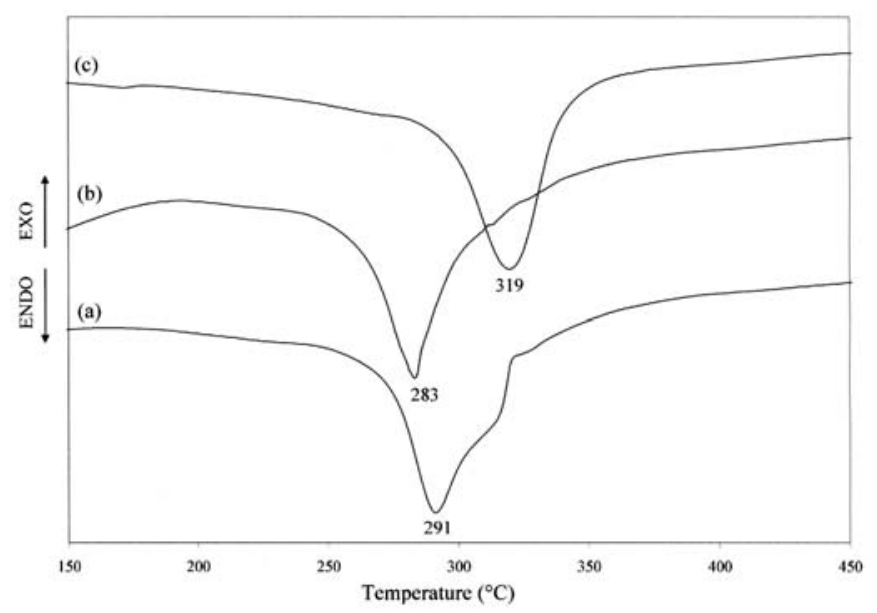

Figure 2. DSC curve of 3D SF-based FA-crosslinked nonwovens showing (a) a bimodal melting/decomposition endotherm with a peak at $291^{\circ} \mathrm{C}$ and a shoulder at $>300^{\circ} \mathrm{C}$. (b) DSC curve of pure crystalline SF film (peak at $283^{\circ} \mathrm{C}$ ). (b) DSC curve of pure $\mathrm{SF}$ fibres (peak at $319^{\circ} \mathrm{C}$ ).

is possible to observe that the fibres are reciprocally stuck at crossover points, with thin films sometimes inlaid amid adjacent fibres (Fig. 1b). These features are attributable to the method used to prepare the nonwovens. In fact, soaking in the $\mathrm{FA}-\mathrm{CaCl}_{2}$ solution caused a partial dissolution of $\mathrm{SF}$ fibres (22). A heterogeneous mixture comprising SF fibres immersed into an acidic SF solution with good film-forming ability (23) was thus obtained. When the solvent was evaporated, soluble SF solidified into a thin film which not only stuck one fibre to another, but even covered some of the smallest interstices among them. The 3D-SF nonwovens used 


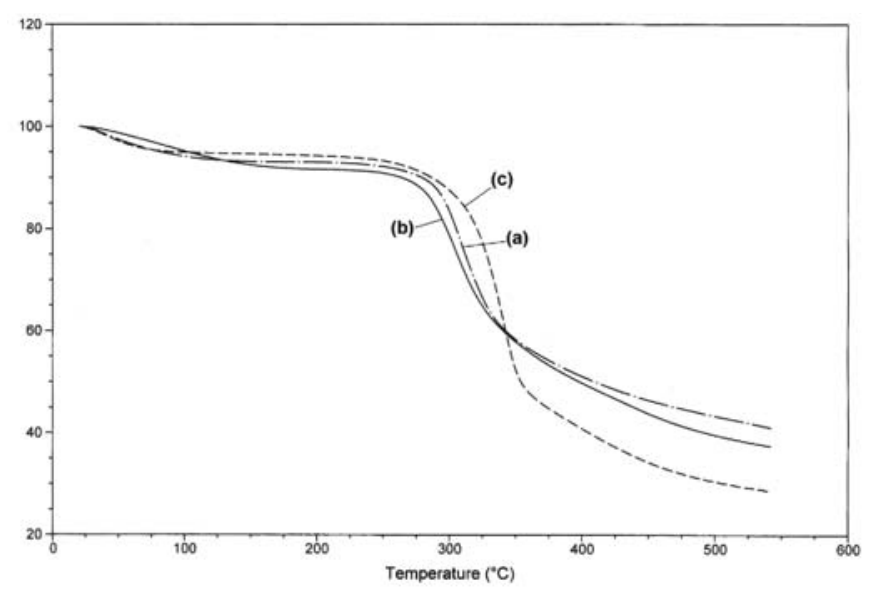

Figure 3. TGA curves of 3D SF-based FA-crosslinked nonwovens (a), of pure crystalline SF film (b), and of pure SF fibres (c). Onset of thermal degradation: (a) $289^{\circ} \mathrm{C}$; (b) $281^{\circ} \mathrm{C}$; and (c) $309^{\circ} \mathrm{C}$.

in this study can be considered a composite material consisting of anisotropic SF fibres incorporated into an isotropic matrix of SF in film form. Both fibres and films are water-insoluble owing to their $\beta$-sheet crystalline structure (20). However, differences in molecular orientation, texture, ductility, and swelling ability of the two components are expected to strongly affect the properties of the resulting composite under dry or wet conditions.

The results of thermal analysis confirmed the composite nature of the 3D-SF nonwovens. The DSC curve (Fig. 2A) is characterised by an intense bimodal endotherm showing the contribution of two components differing in melting/decomposition temperature. The low-temperature component shows a sharp peak at $291^{\circ} \mathrm{C}$, while the high-temperature component exhibits a shoulder at $>300^{\circ} \mathrm{C}$. These components can be attributed to the melting/decomposition of $\beta$-sheet crystalline SF film and fibres, respectively (24). The DSC thermograms of pure SF film (Fig. 2B) and fibres (Fig. 2C), which are characterized by single peaks falling at $283^{\circ} \mathrm{C}$ and $319^{\circ} \mathrm{C}$, respectively, confirm this interpretation. The shift of peak temperature observed for film and fibres in the 3D-SF nonwovens, compared to the reference pure materials, can be related to mutual interactions between the two components in the composite during heating.

Further details on the thermal properties of 3D-SF nonwovens were obtained by TGA analysis (Fig. 3). After an

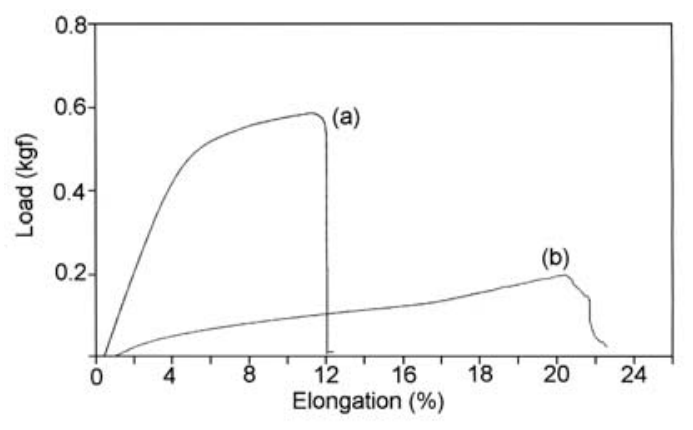

Figure 4. Typical stress-strain curves of 3D SF-based FA-crosslinked nonwovens measured under dry (a) and wet (b) conditions.

initial weight loss due to the evaporation of moisture, 3D-SF nonwovens, SF films, and SF fibres entered a phase characterized by high thermal stability ranging from $100^{\circ} \mathrm{C}$ until $\sim 250^{\circ} \mathrm{C}$, during which the weight remained almost constant. This behaviour is characteristic of crystalline SF materials (25). Afterwards, the weight dropped sharply indicating the onset of extensive thermal degradation caused by SF burning. It is interesting to note that the TGA curve of 3D-SF nonwovens always ran between those of films and fibres, which denotes an intermediate thermal stability, with fibres showing the highest and films showing the lowest. Accordingly, the onset of thermal degradation occurred at increasing temperature values, i.e. at $281^{\circ} \mathrm{C}, 289^{\circ} \mathrm{C}$, and $309^{\circ} \mathrm{C}$, from films to $3 \mathrm{D}-\mathrm{SF}$ nonwoven and fibres, respectively.

Typical stress-strain curves of 3D-SF nonwoven measured under dry and wet conditions are shown in Fig. 4 and results of tensile properties are summarized in Table I. It is important to note that the samples showed a reproducible and uniform mechanical behaviour under whole measuring conditions. 3D-SF nonwovens in the dry state exhibited a curve typical of a stiff material, with an initial region of high strain resistance, a yield point at $\sim 5 \%$ elongation, and a final step of easier extensibility. Likewise for brittle and glassy materials, rupture occurred suddenly across the sample, as indicated by the sharp drop of the curve. The shape of the curve remarkably changed when the sample was preliminarily immersed into water at $37^{\circ} \mathrm{C}$ and tested in the wet state. The initial slope decreased significantly, with a yield point falling at $2 \%$ elongation, after which the sample entered a region characterized by plastic deformation. The change in slope of the stress-strain curve at $\sim 17 \%$ elongation marked the onset of a strain-hardening step.

Table I. Tensile properties of 3D-SF nonwovens measured in the dry and wet state.

\begin{tabular}{lcccc}
\hline Sample & $\begin{array}{c}\text { Strength } \\
(\mathrm{MPa})\end{array}$ & $\begin{array}{c}\text { Elongation } \\
\text { at break }(\%)\end{array}$ & $\begin{array}{c}\text { Modulus } \\
(\mathrm{MPa})\end{array}$ & $\begin{array}{c}\text { Energy } \\
(\mathrm{N} \bullet \mathrm{mm})\end{array}$ \\
\hline Dry & $14.2 \pm 0.8$ & $13.5 \pm 5.2$ & $311.6 \pm 18.2$ & $4.33 \pm 0.63$ \\
Wet & $4.86 \pm 0.55$ & $21.4 \pm 2.5$ & $34.4 \pm 2.7$ & $2.30 \pm 0.32$ \\
SF fibre & $610-690$ & 15 & $15-17 \times 10^{3}$ & $=$ \\
\hline
\end{tabular}

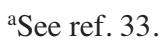


(a)

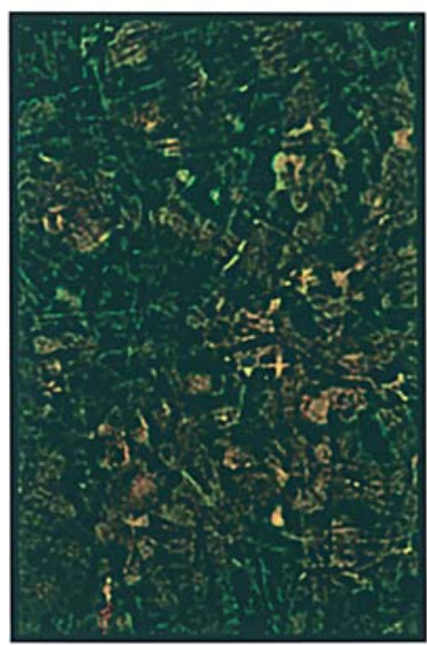

\section{HDFs (green) \\ HEKs (orange-red) \\ Overlaps (yellow)}

(b)

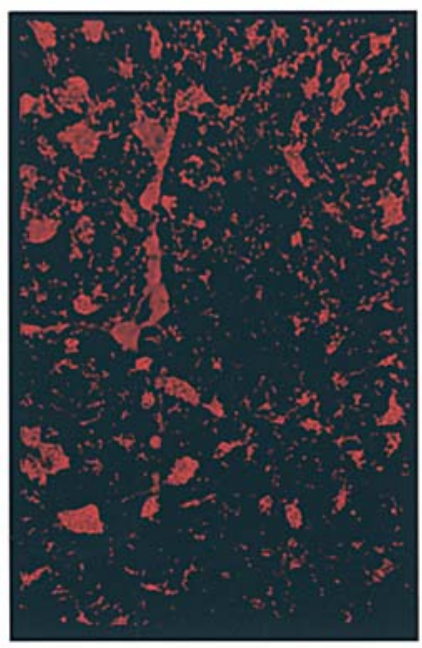

(c)

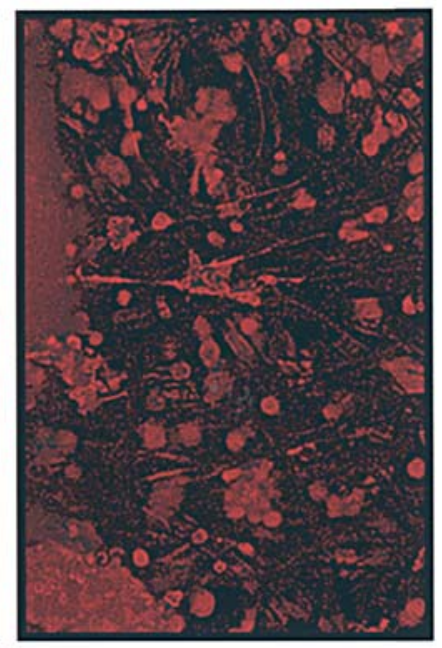

HEKs (red)

Figure 5. Co-cultures of HEKs and HDFs on SF-based, FA-crosslinked 3D nonwovens after approximately 15 days of remaining in vitro. Prior to seeding HDFs were intra-vitally pre-labelled with $\operatorname{DiOC}_{18}(3)$ fluorochrome (green fluorescence, whose intensity peaks at $590 \mathrm{~nm}$ wavelength, when excited by UV light.), whereas HEKs were intra-vitally marked with $\operatorname{DilC}_{18}(3)$ (red fluorescence peaking at 500 nm wavelength, when excited by UV light). Fluorescent micrographs were deconvolved as detailed in the Materials and methods in order to get rid of stray light and thus improve the sharpness of the pictures. (a) The not-too narrow-band FITC optical filter set used here allows observation of not only the HDFs, but even the HEKs, on the SF fibres and their overlapping at some places. (b) and (c) HEKs in clusters of various sizes have adhered to and grown on the SF fibres. The narrow-band rhodamine optical filter set used here does not allow observation of the concurrent presence of the HDFs in the same specimens. Original magnification, x100.

Rupture took place at higher elongation values and was less sharp than for the dried sample. From the results listed in Table I it is possible to observe that, going from the dry to the wet state, strength and modulus decreased by approximately 3- and 9-fold, respectively, while elongation showed a $60 \%$ increase. Accordingly, toughness decreased significantly, as indicated by the value of energy.

The results of tensile measurements indicated that the overall mechanical behaviour of the 3D-SF nonwovens was mainly driven by the properties of the SF component in film form. As evidenced by the reference data listed in Table I, strength and modulus of SF fibres are orders of magnitude larger. Therefore, it is reasonable to suggest that, under the measuring conditions adopted, fibres did not contribute too much to the mechanical resistance of the nonwoven owing to their very short length and random arrangement. Actually, the dramatic change in the stress-strain curve observed in the wet state is indicative of a glassy-to-plastic transformation typical of SF films (26). Absorption of water resulted in extensive swelling of the film, breaking of hydrogen bonds in the less ordered amorphous regions, and easy unfolding of randomly arranged SF chains under straining. The strain-hardening response observed before rupture can be attributed to the stiffening of the film structure owing to orientation of SF chains along the direction of strain and squeezing of water from the film matrix. The final rupture was determined by failure of the film matrix surrounding and holding together the fibres in the composite (27).

These results are of great interest in view of the envisaged tissue engineering/regeneration/repair applications of these 3D
SF-based FA-crosslinked nonwovens. While a certain strength of the material in the dry state may favour its manufacture, handling, and shaping before implantation, the dramatic loss of rigidity under wet conditions renders the same material pliable and mechanically more compliant with the requirements of soft tissues, allowing for a better functional integration of the implanted nonwovens.

Protracted in vitro co-culture of HEKs and HDFs on $3 D$ SFbased FA-crosslinked nonwovens. Most (>90\%) of the seeded HDFs attached to the SF fibres of the 3D nonwovens within $3 \mathrm{~h}$. Twenty-four hours later, the SF-stuck HDFs started proliferating and colonizing the scaffolds as revealed by observations under a fluorescence microscope (Fig. 5). HDFs alone could be cultured and kept growing for at least 15 consecutive weeks onto the nonwoven scaffolds, during which time they exhibited an active metabolism consuming glucose and releasing lactic acid but no urea nitrogen (an index of protein catabolism) into the medium (not shown).

HEKs also rapidly stuck to the SF fibres of the 3D nonwovens that for 10 days had already been bearing the attached HDFs on their opposite site. Initially, SF-fibre-stuck HEKs grew slowly. Yet, 4-6 days later they started forming sizeable patches of cells easily distinguishable for their bright red fluorescence from the green-fluorescing HDFs (Fig. 5). The co-cultured cells steadily metabolised glucose (as well as L-glutamine, not shown) and released lactic acid into the medium for at least 75 days (Fig. 6). Some co-cultures were maintained with no problem for up to 95 days (Fig. 7). While growing, co-cultured HEKs and HDFs came into reciprocal 

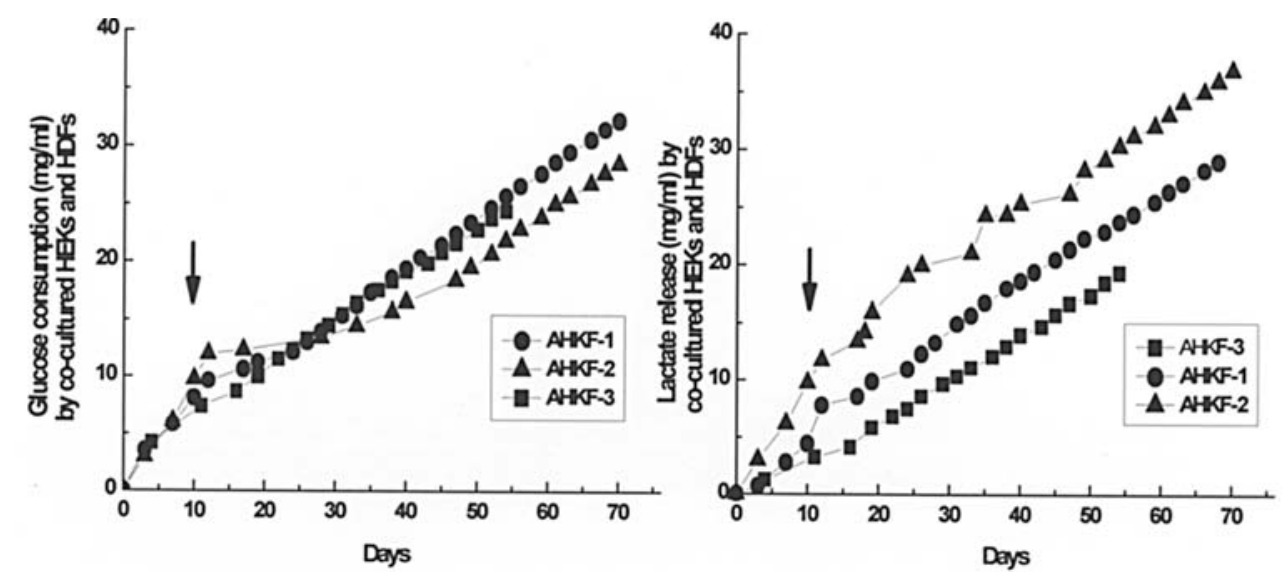

Figure 6. Relevant metabolic activities like glucose consumption (left panel) and lactic acid release (right panel) are carried out steadily for lengthy terms by co-cultured HEKs and HDFs adhering to 3D SF-based FA-crosslinked nonwovens. The cumulative results shown here pertain to three distinct sets (AHKF-1, -2 , and -3) of co-cultured HEKs and HDFs. For technical details consult the Materials and methods. Each point on the curves is the mean value of triplicate determinations. No significant statistical differences occur among the curves of each panel. The arrows indicate the time of HEKs seeding onto nonwovens that on the opposite site had been previously carrying HDFs stuck to their SF fibres.

close contact at several points onto the SF fibres (Fig. 5). Again, the absence of urea nitrogen from the corresponding $\mathrm{HDF} / \mathrm{HEK}$-conditioned growth media (not shown) was proof of the viability and anabolic tendency of the metabolism of co-cultured cells. Notably, during their lengthy stay in vitro, co-cultured HEKs and HDFs never secreted any ELISAassayable amount of the established proinflammatory cytokine IL-1 1 into their growth medium (not shown). Furthermore, in these co-cultures the HDFs retained for 95 days at least some of their highly specific functions, such as the synthesis, release, and extracellular assembly of collagen type-I fibres, as could be assessed by indirectly assaying the amounts of procollagen type-I C-peptide released into the medium (Fig. 7). It should be recalled that, under both normal and pathological conditions, collagen fibres are extracellularly assembled by cutting the C-terminal peptides of the procollagen molecules (28). The unchanging basal rates of assembly of the collagen fibres indicate that the contact with the SF of the nonwovens was not per se fibrogenic.

These results are the first showing that SF-based FAcrosslinked 3D nonwovens, being quite biocompatible with the two main cell types of normal human skin, allow the set up of a novel kind of dermo-epidermal equivalent (29-32). On such scaffolds, keratinocytes and fibroblasts are able to cohabit with reciprocal benefits as they remain viable, metabolically efficient, and capable of performing specific functions for lengthy terms. Thus, our findings are in keeping with the view that SF-based 3D nonwovens are excellent candidates for implant applications in a variety of human clinical settings (e.g., burn wounds, chronic ulcers, etc.) because of their appealing chemico-physical properties and of their superior interactions with normal human skin cells in vitro. This notion is in keeping with the ability of these same scaffolds, once implanted in the animals in vivo, to induce the engineering of a novel, well-vascularised reticular connective tissue without eliciting, even six months after their implantation, an FBR of any significance (20). Further in vitro and animal studies are needed to support the human application attempts of such nonwoven scaffolds. It is conceivable that 3D SF nonwovens may be of use even at sites of implantation other than the skin

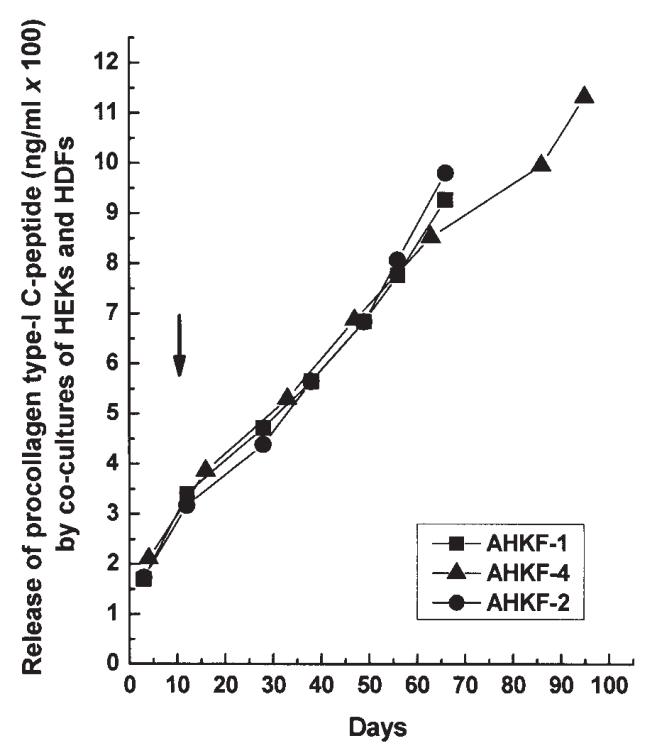

Figure 7. The extracellular assembly of novel collagen fibres occurs steadily for rather lengthy terms on the 3D SF-based FA-crosslinked nonwovens carrying co-cultured of HEKs and HDFs. The cumulative release of the Cpeptide of procollagen type-I was assayed as detailed in the Materials and methods. The results shown here pertain to three distinct sets (AHKF-1, -2, and -4 ) of human HEKs and HDFs. Each point on the curves is the mean value of triplicate determinations. The arrow indicates the time of HEKs seeding onto nonwovens that on their opposite site had been previously carrying HDFs stuck to their SF fibres. In one instance, i.e. the AHKF-4 coculture set, the sampling was carried out up to the 95th day in vitro; in the other two instances it was instead devised to terminate the co-cultures at the 65 th day.

(such as bone, cartilage, tendons, etc.). It also goes without saying that it will be necessary to optimise the structure of the 3D nonwovens according to the specific requirements of each likely site of application. Moreover, it will be essential to take heed of ongoing technological progress. Hopefully in a not so distant future, SF-based 3D nonwoven scaffolds made of microfibres or nanofibres (33-35) or both will suit quite different goals in the exciting fields of human tissue engineering, regeneration, and repair. 


\section{Acknowledgements}

The authors wish to express their gratitude to Mr. Walter Carrer of Stazione Sperimentale per la Seta (Milan, Italy) for his contribution to the tensile measurements.

\section{References}

1. Zhou CZ, Confalonieri F, Medina N, Zivanovic Y, Esuault C, Yang T, Jacquet M, Janin J, Duguet M, Perasso R and Li ZG: Fine organization of Bombyx mori fibroin heavy chain gene. Nucleic Acids Res 28: 2413-2419, 2000.

2. Soong HK and Kenyon KR: Adverse reactions to virgin silk sutures in cataract surgery. Ophthalmology 91: 479-483, 1984.

3. Wen CM, Ye ST, Zhou LX and Yu Y: Silk-induced asthma in children: a report of 64 cases. Ann Allergy 65: 375-378, 1990.

4. Kurosaki S, Otsuka H, Kunitomo M, Koyama M, Pawankar R and Matumoto K: Fibroin allergy: IgE mediated hypersensitivity to silk suture materials. Nippon Ika Daigaku Zasshi 66: 41-44, 1999.

5. Rossitch E Jr, Bullard DE and Oakes WJ: Delayed foreign-body response to silk sutures in pediatric neurosurgical patients. Childs Nerv Syst 3: 375-378, 1987.

6. Setzen G and Williams EF: Tissue response to suture materials implanted subcutaneously in a rabbit model. Plast Reconstruct Surg 100: 1788-1795, 1997.

7. Nebel L, Rosenberg G, Tobias B and Nathan H: Autograft suture in peripheral nerves. Eur Surg Res 9: 224-234, 1977.

8. Sakabe H, Ito H, Miyamoto T, Noishiki Y and Ha WS: In vivo blood compatibility of regenerated silk fibroin. Sen-I Gakkaishi 45: 487-490, 1989.

9. Santin M, Motta A, Freddi G and Cannas M: In vitro evaluation of the inflammatory potential of the silk fibroin. J Biomed Mater Res 46: 382-389, 1999.

10. Chiarini A, Petrini P, Bozzini S, Dal Pra I and Armato U: Silk fibroin/poly(carbonate)-urethane as a substrate for cell growth: in vivo interactions with human cells. Biomaterials 24: 789-799, 2003.

11. Dal Pra I, Petrini P, Chiarini A, Bozzini S, Fare S and Armato U: Silk fibroin-coated three-dimensional polyurethane scaffolds for tissue engineering: interactions with normal human fibroblasts. Tissue Eng 9: 1113-1121, 2003.

12. Minoura N, Tsukada M and Nagura M: Physico-chemical properties of silk fibroin membrane as a biomaterial. Biomaterials 11: 430-434, 1990.

13. Minoura N, Aiba S, Gotoh Y, Tsukada M and Imai Y: Attachment and growth of cultured fibroblast cells on silk protein matrices. J Biomed Mater Res 29: 1215-1221, 1995.

14. Gotoh Y, Tsukada M and Minoura N: Effect of the chemical modification of the arginyl residue in Bombyx mori silk fibroin on the attachment and growth of fibroblast cells. J Biomed Mater Res 39: 351-357, 1998.

15. Inouye $K$, Kurokawa $M$, Nishikawa $S$ and Tsukada $M$ : Use of Bombyx mori silk fibroin as a substratum for cultivation of animal cells. J Biochem Biophys Methods 37: 159-164, 1998.

16. Norihiko M, Sei-ichi A, Yohko G, Masuhiro T and Yohji I: Attachment and growth of cultured fibroblast cells on silk fibroin matrices. J Biomed Mater Res 29: 1215-1221, 1995.
17. Armato U, Migliaresi C and Motta A: Bio-artificial substrate for the production of animal and, in particular, human tissue and organs. Patent PTC WO 01/25403 A3, published September 28, 2001 .

18. Sofia S, McCarthy MB, Gronowicz G and Kaplan DL: Functionalized silk-based biomaterials for bone formation. J Biomed Mater Res 54: 139-148, 2001.

19. Altman GH, Diaz F, Jakuba C, Calabro T, Horan RL, Chen J, Lu H, Richmond J and Kaplan DL: Silk-based biomaterials. Biomaterials 24: 401-416, 2003.

20. Dal Pra I, Freddi G, Minic J, Chiarini A and Armato U: De novo engineering of reticular connective tissue in vivo by silk fibroin nonwoven materials. Biomaterials 26: 1987-1999, 2005.

21. Armato U, Dal Pra I, Kesenci K, Migliaresi C and Motta A: Method for the preparation of non-woven silk fibroin fabrics. Patent PTC WO 02/29141 A1, published April 11, 2002.

22. Earland C and Raven JR: A new solvent for silk. Nature 44: $461,1954$.

23. Asakura T, Yao J, Yamane T, Umemura K and Ulrich AS: Heterogeneous structure of silk fibres from Bombyx mori resolved by $13 \mathrm{C}$ solid-state NMR spectroscopy. J Am Chem Soc 124: 8794-8795, 2002.

24. Arai T, Freddi G, Innocenti R and Tsukada M: Preparation of water repellent silks by a reaction with octadecenylsuccinic anhydride. J Appl Polym Sci 89: 324-332, 2003.

25. Lee SM, Cho DH, Park WH, Lee SG, Han SO and Drzal LT: Novel silk/poly(butylenes succinate) biocomposites: the effect of short fibre on their mechanical and thermal properties. Compos Sci Technol 65: 647-657, 2005.

26. Minoura M, Tsukada M and Nagura M: Physico-chemical properties of silk fibroin membrane as a biomaterial. Biomaterials 11: 430-434, 1990.

27. Perez-Rigueiro J, Viney C, Llorca J and Elices M: Mechanical properties of single-brim silkworm silk. J Appl Polym Sci 75: 1270-1277, 2000.

28. Duncan MR and Berman B: Stimulation of collagen and glycosaminoglycan production in cultured human adult dermal fibroblasts by recombinant human interleukin-6. J Invest Dermatol 97: 686-692, 1991.

29. Konig A and Bruckner-Tuderman L: Transforming growth factor-beta promotes deposition of collagen VII in a modified organotypic skin model. Lab Invest 70: 203-209, 1994.

30. Dubertret L: Reconstruction of the human skin equivalent in vitro: a new tool for skin biology. Skin Pharmacol 3: 144-148, 1990.

31. Shahabeddin L, Berthod F, Damour O and Collombel C: Characterization of skin reconstructed on a chitosan-cross-linked collagen-glycosaminoglycan matrix. Skin Pharmacol 3: 107-114, 1990 .

32. Tinois E, Faure M, Chatelain P, Vallier P and Schmitt D: Growth and differentiation of human keratinocytes on extracellular matrix. Arch Dermatol Res 279: 241-246, 1987.

33. Altman GH, Horal RL, Lu HH, Moreau J, Martin I, Richmond JC and Kaplan DL: Silk matrix for tissue engineered anterior cruciate ligaments. Biomaterials 23: 4131-4141, 2002.

34. Jin H-J, Chen J, Karageorgiou V, Altman GH and Kaplan DL: Human bone marrow stromal cells responses on electrospun silk fibroin mats. Biomaterials 25: 1039-1047, 2004.

35. Min B-M, Lee G, Kim SH, Nam YS, Lee TS and Park WH: Electrospinning of silk fibroin nanofibers and its effect on the adhesion and spreading of normal human keratinocytes and fibroblasts in vitro. Biomaterials 25: 1289-1297, 2004. 